\title{
An Investigation into Time-Domain Approach for OFDM Channel Estimation
}

\author{
Hlaing Minn, Student Member and Vijay K. Bhargava, Fellow, IEEE
}

\begin{abstract}
A time-domain based channel estimation for OFDM system with pilot-data multiplexed scheme is investigated. As an approximation to linear minimum mean square estimator (LMMSE), a time-domain based channel estimation is proposed where intra-symbol time-averaging and most significant channel taps selection are applied. The relation and differences of the proposed method to DFT-based LMMSE methods are discussed. The performances of the proposed method, DFT-based LMMSE method [15] and the methods of [16] and [17] are evaluated in multipath fading channels. The simulation results show that proposed method achieves almost the same performance as DFT-based LMMSE method and better BER performance than the other methods while keeping less complexity.
\end{abstract}

Index Terms-Channel estimation, intra-symbol time-averaging, linear minimum mean square error estimator, most significant taps selection, orthogonal frequency division multiplexing (OFDM).

\section{INTRODUCTION}

$\mathbf{O}$ RTHOGONAL frequency division multiplexing (OFDM) [1]-[3] has recently achieved much popularity due to its desirable properties such as its robustness to multipath delay spread and impulse noise, its high data rate transmission capability with high bandwidth efficiency, and its feasibility in application of adaptive modulation and power allocation across the subcarriers according to the channel conditions. It has been adopted in wireline applications such as ADSL (Asymmetric Digital Subscriber Line) [4], broadcasting services such as European DAB (Digital Audio Broadcasting) [5], DVB-T (Terrestrial Digital Video Broadcasting) [6] and Japanese ISDB-T (Terrestrial Integrated Services Digital Broadcasting) [7], high rate wireless LAN standards such as ETSI HiperLAN 2 and IEEE 802.11(a), and multimedia wireless services such as Japanese MMAC (Multimedia Mobile Access Communications) [8], [9].

If noncoherent OFDM system is used, the system complexity will be reduced at the cost of 3-4 dB performance loss [10]. If coherent OFDM system is adopted, channel estimation becomes a requirement and usually pilot tones are used for channel (frequency response) estimation. Pilot tones can be inserted in all subcarriers of a particular OFDM symbol forming an OFDM training symbol, in which case training symbols are transmitted at an appropriate regular rate determined by the time varying nature of the wireless channel. Another approach is that, instead of using all subcarriers, the pilot tones are multiplexed

Manuscript received October 24, 2000. This work was supported in part by a Strategic Project Grant from the Natural Sciences and Engineering Research Council (NSERC) of Canada and in part by Telus Mobility, Cellular.

The authors are with the Department of Electrical and Computer Engineering, University of Victoria, Victoria, B.C., Canada V8W 3P6.

Publisher Item Identifier S 0018-9316(00)11686-5. with data to form OFDM symbols. The subcarrier spacing between pilot tones is usually determined by the frequency selectivity of the wireless channel. The pilot multiplexing can be allowed for all OFDM symbols (i.e., all the time of transmission) or at an appropriate rate depending on the time selectivity of the wireless channel. In channel estimation using training symbols, decision directed approach has to be used. If complexity is affordable, time interpolation (e.g., [11]) can be used to improve the performance. If pilot tone multiplexing is used, the frequency interpolation has to be performed [12]-[14]. Similarly, time domain interpolation can be performed at the cost of complexity. Most of the channel estimation approaches may be viewed as DFT-based approaches [15], [16], [10], [11], where LS (Least square) channel (frequency response) estimates are fed to IFFT block to get time domain channel impulse response estimate, and then appropriately processed and transformed back to frequency domain by FFT. A DFT-based approach for OFDM system with transmit and receive antenna diversity has been discussed in [10].

In this paper, we investigate a time-domain channel estimation approach, namely FPTA (Frequency Pilot Time Average) [17] which applies intra-symbol time-domain averaging of identical parts of the pilot signal. We also propose a time-domain approach for OFDM channel estimation which achieves performance gain over LS or FPTA approaches.

The rest of the paper is organized as follows. In Section II, the OFDM system and LS channel estimation are presented for notational description. The method of time-averaging the identical parts of a pilot signal is briefly presented in Section III, and our analysis on this intra-symbol time-averaging is given in Section IV. Section V presents the proposed time-domain channel estimation and its relation to DFT-based approaches are discussed in Section VI. Simulation results are discussed in Section VII and conclusions are given in Section VIII.

\section{SYSTEM DESCRIPTION}

Suppose the pilot tones $P[k]$ are multiplexed with data $D[k]$ in all OFDM symbols at a pilot ratio $1 / K$ (ratio of the number of pilot tones to the total number of subcarriers) where $k$ is subcarrier index $(0,1, \cdots, N-1)$ with $N$ being the total number of subcarriers, and $P[k]$ and $D[k]$ are zeros except at their corresponding subcarriers. Then the transmitted OFDM signal in discrete-time domain, excluding guard-interval, can be expressed as

$$
\begin{aligned}
& s[n]=I F F T_{N}\{P[k]\}+I F F T_{N}\{D[k]\} \\
& =p[n]+d[n]
\end{aligned}
$$


where $I F F T_{N}\{\}$ is $N$-point inverse Fast Fourier transform and $n$ is the time-domain index $(0,1, \cdots, N-1)$ of an OFDM symbol. Suppose the wireless channel has a discrete-time impulse response given by

$$
\left.h[n]=\sum_{l=0}^{L-1} \alpha_{l} \delta\left[n-\tau_{l}\right]\right)
$$

where

$\alpha_{l} \quad$ is complex path gain of $l$ th path,

$\tau_{l} \quad$ is the delay of $l$ th path, and

$L \quad$ is the total number of channel paths.

For simplicity, time dependence nature of the channel impulse response is suppressed in the notation.

After passing through a multipath wireless channel, the timedomain received samples of an OFDM symbol, if appropriate cyclic prefix guard samples are used, is given by

$$
r[n]=s[n] \otimes h[n]+w[n]
$$

where $\otimes$ represents $N$-point circular convolution, $\{w[n]\}$ are independent and identically distributed (iid) AWGN samples with zero mean and variance of $\sigma_{t}^{2}$. Assuming perfect synchronization, the FFT output frequency-domain subcarrier symbols can be expressed as

$$
\begin{aligned}
R[k]= & F F T_{N}\{r[n]\}=H[k] P[k] \\
& +H[k] D[k]+W[k]
\end{aligned}
$$

where $W[k]=F F T_{N}\{w[n]\}$ is frequency-domain AWGN noise samples with zero mean and variance $\sigma_{f}^{2}=N \sigma_{t}^{2}$. Then the channel frequency response at the pilot tones can be estimated by

$$
\hat{H}[m]=\frac{R[m]}{P[m]}=H[m]+\frac{W[m]}{P[m]}
$$

where $m$ is the subcarrier index for pilot tones. This channel estimate is called LS (least square) estimate. The channel responses at other subcarriers can be obtained by interpolation.

\section{Frequency Pilot Time Average (FPTA) Method}

In FPTA [17] approach, positive and negative alternatively polarized pilot tones are multiplexed with data at a pilot ratio of $1 / K$. The frequency-domain pilot tones can be expressed as

$$
P[k]= \begin{cases}(-1)^{m} A, & k=K m \\ 0, & k=K m+i\end{cases}
$$

where

$K, m$ and $i \quad$ are integers, $1 \leq i \leq K-1,0 \leq m \leq M-1$, $A \quad$ is pilot amplitude and

$M=N / K \quad$ is an integer.

The corresponding time-domain pilot samples can be expressed as

$$
\begin{aligned}
p[n] & =I F F T_{N}\{P[k]\}=\frac{1}{N} \sum_{k=0}^{N-1} P[k] W_{N}^{-n k} \\
& =\frac{A}{K} \sum_{r=0}^{K-1} \delta[n-M r-M / 2]
\end{aligned}
$$

where $W_{N}=\exp (-j 2 \pi / N)$.
In FPTA approach, since there are $K$ identical parts of timedomain pilot samples, the corresponding parts of received samples are averaged over $K$ parts. This intra-symbol time-domain averaging reduces the variance of noise samples by $K$ times, i.e., $\sigma_{t, a v g}^{2}=\sigma_{t}^{2} / K$. Based on this observation, [17] defined time-domain pilot to noise ratio as $p[n] / \sigma_{t, a v g}$ and compared it with frequency domain pilot to noise ratio $P[k] / \sigma_{f}$ resulting in the following expression

$$
\frac{p[n] / \sigma_{t, a v g}}{P[k] / \sigma_{f}}=\sqrt{\frac{N}{K}}
$$

which was mentioned as the gain of FPTA approach over frequency domain approach such as LS estimation discussed in Section II. However, this result is optimistic and more details on it will be discussed in the following section.

\section{AnAlysis of InTRA-Symbol Time AVERAGING IN FPTA}

In order to investigate the pilot to noise ratio insightfully, we neglect the data part and consider only the pilot affected by multipath channel and Gaussian noise. Then the time-domain received samples vector of an OFDM signal can be given by

$$
\mathbf{r}=\mathbf{p}^{\prime}+\mathbf{w}
$$

where $\mathbf{r}=\left[\mathrm{r}_{0}, \mathbf{r}_{1}, \cdots, \mathbf{r}_{K-1}\right]$ with $\mathbf{r}_{i}=\left[r_{i}[0]\right.$, $\left.r_{i}[1], \cdots, r_{i}[M-1]\right], \mathbf{p}^{\prime}$ is $N$-point circular convolution of pilot signal $\mathbf{p}$ and channel impulse response $\mathbf{h}$ and can be expressed as $\mathbf{p}^{\prime}=\left[\mathbf{p}_{0}^{\prime}, \mathbf{p}_{1}^{\prime}, \cdots, \mathbf{p}_{K-1}^{\prime}\right]$ with $\mathbf{p}_{i}^{\prime}=\left[p_{i}^{\prime}[0]\right.$, $\left.p_{i}^{\prime}[1], \cdots, p_{i}^{\prime}[M-1]\right]$ and $\mathbf{w}=[w[0], w[1], \cdots, w[N-1]]$ is iid Gaussian noise samples.

Then averaging the received samples over $K$ parts, assuming the channel impulse response is constant over the OFDM symbol interval, gives

$$
\mathbf{r}_{a v g}=\frac{1}{K} \sum_{l=0}^{K-1} \mathbf{r}_{l}=\mathbf{p}_{0}^{\prime}+\mathbf{w}_{a v g}
$$

where $\mathbf{w}_{\text {avg }}=\left[w_{\text {avg }}[0], w_{\text {avg }}[1], \cdots, w_{\text {avg }}[M-1]\right]$ with $w_{\text {avg }}[i]=(1 / K) \sum_{j=0}^{K-1} w[i+j M]$. Since $\{w[n]\}$ are iid zero mean complex Gaussian random variables with variance $\sigma_{t}^{2},\left\{w_{\text {avg }}[i]\right\}$ are iid zero mean complex Gaussian random variables with variance $\sigma_{t, a v g}^{2}=\sigma_{t}^{2} / K$.

Now, we investigate two possible approaches for channel estimation using intra-symbol time-averaged received samples. The first approach, which will be denoted by FPTA-1 in the rest, is to reconstruct the received samples of length $N$ by repeating the $\mathbf{r}_{\text {avg }}, K$ times. Then the reconstructed samples $\mathbf{y}$ can be expressed as

$$
\mathbf{y}=\left[\mathbf{r}_{a v g}, \mathbf{r}_{a v g}, \cdots, \mathbf{r}_{a v g}\right]=\mathbf{p}^{\prime}+\mathbf{v}
$$

where $\mathbf{v}=\left[\mathbf{w}_{\text {avg }}, \mathbf{w}_{\text {avg }}, \cdots, \mathbf{w}_{\text {avg }}\right]$ is $K$ times repeated version of $\mathbf{w}_{\text {avg }}$. The corresponding frequency domain samples are

$$
Y[k]=F F T_{N}\{y[n]\}=H[k] P[k]+V[k]
$$

where $\{P[k]\}$ are original pilot tones, $H[k]$ is channel frequency response and $V[k]=F F T_{N}\{\mathbf{v}\}$ is frequency-domain zero 
mean Gaussian noise term. The channel frequency responses at pilot tones can be estimated by

$$
\hat{H}[m]=Y[m] / P[m]=H[m]+V[m] / P[m]
$$

where $m=0, K, 2 K, \cdots,(M-1) K$.

The variance of $V[m]$ can be given as follows:

$$
\begin{aligned}
\operatorname{var}\{V[m]\}= & E\left\{V[m] V^{*}[m]\right\}, \\
& m=0, K, 2 K, \cdots,(M-1) K \\
= & E\left\{\sum_{i=0}^{N-1} v[i] W_{N}^{m i} \sum_{j=0}^{N-1} v^{*}[j] W_{N}^{-m j}\right\} \\
= & E\left\{\sum_{i=0}^{M-1} v[i] \sum_{l=0}^{K-1} W_{N}^{m(i+l M)} \sum_{j=0}^{M-1} v^{*}[j]\right. \\
& \left.\cdot \sum_{n=0}^{K-1} W_{N}^{-m(j+n M)}\right\} \\
= & \sum_{i=0}^{M-1} \sum_{j=0}^{M-1} E\left\{v[i] v^{*}[j]\right\} \sum_{l=0}^{K-1} W_{N}^{m(i+l M)} \\
& \cdot \sum_{n=0}^{K-1} W_{N}^{-m(j+n M)} \\
= & \sum_{i=0}^{M-1} \sigma_{t, a v g}^{2}\left|\sum_{l=0}^{K-1} W_{N}^{m(i+l M)}\right|^{2} \\
= & \sum_{i=0}^{M-1} \sigma_{t, a v g}^{2} K^{2}=N \sigma_{t}^{2}
\end{aligned}
$$

which is the same as the variance of frequency-domain noise term without intra-symbol time-averaging (i.e., $W[k]$ in (4)). The pilot to noise ratio is also the same, given by $A / \sqrt{N \sigma_{t}^{2}}$.

The second possible approach, which will be denoted by FPTA-2 in the rest, is to use $F F T_{N}\left\{\mathrm{r}_{\text {avg }}\right\}$ together with $F F T_{N}\left\{\mathbf{p}_{0}\right\}$ where $\mathbf{p}_{0}$ is one of the identical parts of time-domain pilot samples. The channel frequency response is estimated as follows:

$$
\begin{aligned}
P_{0}[k]= & F F T_{N}\left\{p_{0}[n]\right\}=A / K, \\
& \quad \text { for } k=0,1, \cdots, N-1 \\
R_{\text {avg }}[k]= & F F T_{N}\left\{r_{\text {avg }}[n]\right\}=H[k] P_{0}[k]+W_{\text {avg }}[k] \\
\hat{H}[k]= & R_{\text {avg }}[k] / P_{0}[k]=H[k]+W_{\text {avg }}[k] / P_{0}[k]
\end{aligned}
$$

where $\left\{W_{\text {avg }}[k]\right\}$ are zero mean complex Gaussian noise terms and $\left\{P_{0}[k]\right\}$ are the equivalent pilot tones for this approach. It can be shown that the variance of $W_{\text {avg }}[k]$ is $M \sigma_{t, a v g}^{2}(=$ $\left.(M / K) \sigma_{t}^{2}\right)$. The pilot to noise ratio is the same as the previous case.

The channel estimation error is given by $e_{H}=\hat{H}[k]-H[k]$ which is the second term in $\hat{H}[k]$ equation. Since its mean is zero, the mean square error (mse) of channel estimate, $m s e\{\hat{H}[k]\}=E\left[e_{H} e_{H}^{*}\right]$, is equal to the variance of the second term. Hence the channel estimation mse's of LS method and intra-symbol time-averaging methods are the same and equal to $N \sigma_{t}^{2} / A^{2}$.

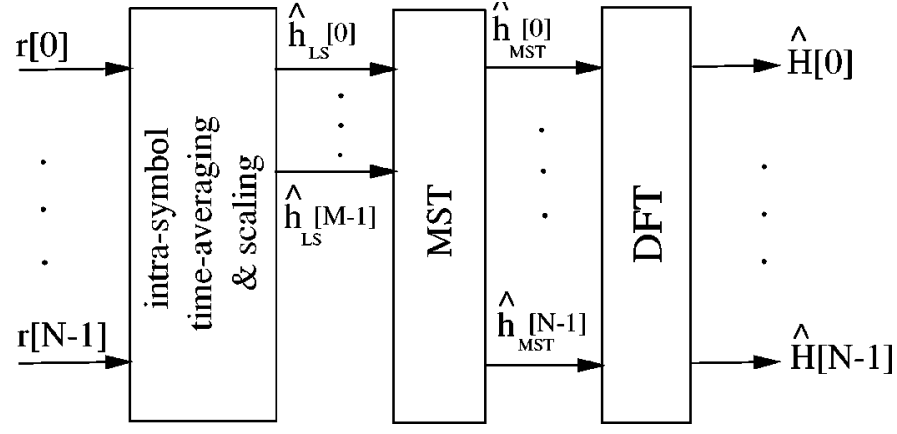

Fig. 1. Most Significant Taps (MST) Method.

The above discussion also indicates that intra-symbol timeaveraging alone does not achieve channel estimation performance gain and the result in (8) is optimistic. Another way of explanation for the result in (8) is that it considers only one time-domain sample (after averaging) while other samples also affect the channel estimate. The result would be appropriate if $p[n] / \sigma_{t, a v g}$ in (8) is obtained by averaging over all $M$ samples (after averaging). In the rest, the gain of a channel estimation method over another (if not mentioned, LS method is assumed) will be expressed by the mse gain (i.e., the ratio of $m s e$ 's) or the (frequency-domain) pilot-to-noise power ratio instead of the pilot amplitude to noise standard deviation ratio.

\section{Most Significant TAPS APPROACH}

For practical multipath wireless channels, there are not so many channel paths with significant energy (if compared to the FFT size $N$ ). Hence, among $N$ samples (taps) of the channel impulse response estimate, many samples (taps) will have little or no energy at all except noise perturbation. Neglecting those nonsignificant channel taps in channel estimation may introduce some performance degradation if some of the channel energy is missed, but at the same time it will eliminate the noise perturbation from those taps. Usually total noise perturbation from those neglected channel estimate taps is much higher than the multipath energy contained in them, especially for low SNR values. Hence, neglecting those nonsignificant channel estimate taps can improve the channel estimation performance significantly and this fact is applied in the proposed method as shown in Fig. 1.

We consider the same scenario as in [17] where pilot tones are multiplexed with data in each OFDM symbol at a pilot ratio of $1 / K$. The pilot tone used in the proposed $M S T$ approach is

$$
P[k]=\sum_{m=0}^{M-1} A \delta[k-m K], \quad k=0,1, \cdots, N-1
$$

and the corresponding time-domain samples contain $K$ identical parts and are given by

$$
p[n]=\sum_{m=0}^{K-1} \frac{A}{K} \delta[n-m M], \quad n=0,1, \cdots, N-1
$$

If the maximum channel delay spread is less than the length of an identical part, which can be designed to satisfy this, then the time-domain received samples corresponding to the timedomain pilot samples contain $K$ parts, each representing a 
scaled channel impulse response for the respective part corrupted by AWGN. If the channel path gains remain essentially the same over an OFDM symbol interval, which is usually the case since OFDM systems are usually designed to satisfy this in order to maintain orthogonality among subcarriers, then the received samples corresponding to time-domain pilot samples contain $K$ repeated version of scaled channel impulse response which are independently corrupted by AWGN. In order to choose most significant channel taps, those $K$ parts can be averaged so that the noise variance is reduced by $K$ times and more reliable most significant channel taps can be obtained. In mathematical expression, the time-domain received samples corresponding to time-domain pilot samples, (For simplicity, data part is neglected in the received samples expression. The mean of data part after averaging is zero), can be given by

$$
\begin{aligned}
r[n] & =h[n] \otimes p[n]+w[n] \\
& =\frac{A}{K} \sum_{m=0}^{K-1} h[n-m M]+w[n], \\
& n=0,1, \cdots, N-1
\end{aligned}
$$

After averaging, we have the noise-corrupted scaled channel impulse response

$$
r_{\text {avg }}[n]=\frac{A}{K} h[n]+w_{\text {avg }}[n], \quad n=0,1, \cdots, M-1
$$

Then the raw channel impulse response estimate is given by

$$
\begin{gathered}
\hat{h}_{L S}[n]=\frac{K}{A} r_{a v g}[n]=h[n]+\frac{K}{A} w_{\text {avg }}[n], \\
n=0,1, \cdots, M-1
\end{gathered}
$$

Now, the most significant $J$ channel taps are chosen as the $J$ largest amplitude channel taps. Let the channel tap indexes for those most significant $J$ taps be denoted by $n_{0}, n_{1}, \cdots, n_{J-1}$. Then the time-domain channel impulse response estimate of proposed MST method is obtained by setting the other channel tap gains to zero as shown below:

$$
\begin{array}{r}
\hat{h}_{M S T}[n]=\sum_{i=0}^{J-1} \hat{h}_{L S}\left[n_{i}\right] \delta\left[n-n_{i}\right], \\
n=0,1, \cdots, N-1
\end{array}
$$

The channel frequency response estimate is directly obtained by applying $F F T$ to $\left\{\hat{h}_{M S T}[n]\right\}$ as

$$
\begin{aligned}
\hat{H}_{M S T}[k] & =F F T_{N}\left\{\hat{h}_{M S T}[n]\right\}, \quad k=0,1, \cdots, N-1 \\
& =H[k]-H_{\text {res }}[k]+W_{\text {avg }}[k]
\end{aligned}
$$

where

$$
\begin{aligned}
H[k] & =\sum_{n=0}^{N-1} h[n] W_{N}^{k n} \\
H_{\text {res }}[k] & =\sum_{l=0}^{N-J-1} h\left[n_{l}^{\prime}\right] W_{N}^{k n_{l}^{\prime}} \\
W_{\text {avg }}[k] & =\frac{K}{A} \sum_{i=0}^{J-1} w_{\text {avg }}\left[n_{i}\right] W_{N}^{n_{i} k}
\end{aligned}
$$

with $\left\{n_{i}\right\}$ being the most significant channel tap indexes and $\left\{n_{l}^{\prime}\right\}$ being the indexes of the other channel taps.

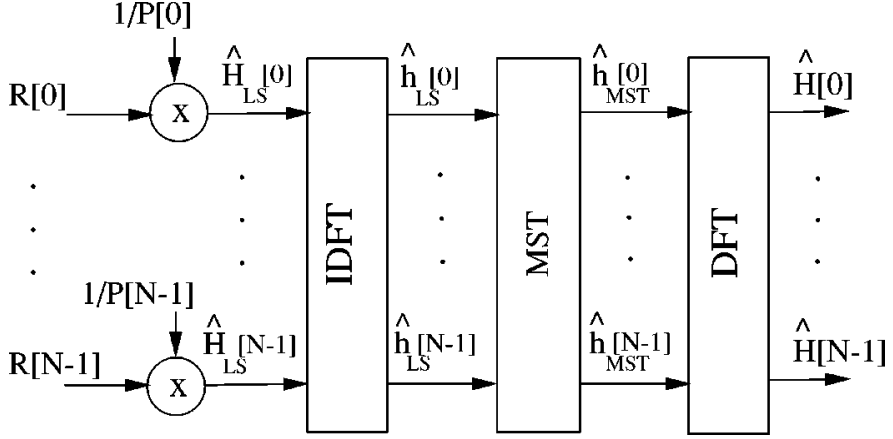

Fig. 2. MST's dual form DFT-based method.

By using suitable number of most significant taps, $H_{\text {res }}[k]$ can be kept very small (or zero) and hence the channel estimation error is mostly dominant (or totally caused) by the noise term $W_{\text {avg }}[k]$. For this case, the mse of the channel estimate can be approximated (or expressed) as

$$
\begin{aligned}
\operatorname{mse}\{\hat{H}[k]\} & =E\left\{W_{\text {avg }}[k] W_{\text {avg }}^{*}[k]\right\}=\frac{K^{2}}{A^{2}} J \sigma_{t, a v g}^{2} \\
& =\frac{K J \sigma_{t}^{2}}{A^{2}}=\frac{K J}{N} m \operatorname{se}\left\{\hat{H}_{L S}[k]\right\}
\end{aligned}
$$

Hence, the mse performance gain of proposed MST method over LS method (similarly over FPTA method) is ideally $N / J K$. The actual mse performance gain would be less than this amount due to the interference from data part and some (if any) excluded channel taps of nonsignificant energy.

The choice of the number of most significant taps $J$ in the channel estimation depends on the application scenario. Broadcasting environment such as single frequency network can have larger number of multipaths with significant energy than nonbroadcasting cases such as wireless LAN's environment. In any case, $J$ should be chosen larger than the (designed) number of multipaths in order to prevent channel estimation error caused by missed channel taps. The channel estimation error caused by the noise from an additional tap in channel estimation is much less than that caused by missing one of the multipaths. A suitable choice for $J$ may be two times or more of the (designed) number of multipaths (as will be seen in the simulation results) in order to ensure no channel energy missing.

Another MST tap selection approach can be implemented by selecting the channel taps whose energy is above a threshold. The threshold may be set as $\eta$ times the maximum channel tap's energy in the raw channel estimate. The suitable choice of $\eta$ depends on the operating SNR and more details will be discussed in the simulation results section.

\section{Similar ApProaches IN DFT-BASEd Methods}

In this section, we relate the proposed MST method to the DFT-based approaches [15], [16], [10]. Essentially, due to the one-to-one relationship of DFT and IDFT, the MST method can be related to DFT-based approach as shown in Fig. 2. Consider the system with training symbol (i.e., pilot tones on all subcarriers). First, LS estimates are obtained and then input to IFFT block resulting in $N$ samples LS estimate of channel impulse response. The largest amplitude $J$ channel taps among the $N$ samples (taps) are chosen as $J$ most significant channel taps and 
the other taps are set to zero. The resulting MST channel impulse response estimate is input to FFT block to get the MST channel frequency response estimate. This MST's dual form DFT-based approach has been applied in [10]. A similar concept by using singular value decomposition can be found in [18].

The difference between proposed MST approach and its dual form DFT-based approach is that MST uses pilot-data multiplexed approach while its dual form DFT based approaches use training symbol approach. It can be shown that the potential gain of the latter approach is $N / J$. Using total pilot power of $N A^{2}$, the latter approach achieves potential gain of $N / J$ whereas MST achieves potential gain of $N /(J K)$ with total pilot power of $N A^{2} / K$; hence, on the basis of the same total pilot power, both methods have the same potential gain for channel estimation. Another difference is the complexity. In MST approach, operations involved are time-averaging, most significant channel taps selection and one FFT operation whereas its dual form DFT-based approach requires LS estimation, one IFFT operation, most significant channel taps selection and one FFT operation. Hence, the proposed MST approach saves some complexity.

One approach which is similar to MST's dual form DFTbased approach is the method of [16]. The difference is that [16] uses the first $N_{c p}$ channel taps where $N_{c p}$ is the number of cyclic prefix samples whereas MST's dual form DFT-based approach uses only $J$ most significant channel taps, $\left(J<N_{c p}\right)$. Since multipath channels usually have much less channel taps than $N_{c p}$, [16] has more noise perturbation than MST.

Other DFT-based approaches use linear minimum mean square error (LMMSE) estimators or approximate LMMSE estimators with reduced complexity [15]. If complexity can be afforded, these LMMSE or approximate LMMSE estimators can be implemented in MST approach. Let us consider a wide-sense stationary uncorrelated scattering (WSSUS) multipath channel with power delay profile given by $\sigma_{h}^{2}$ at delays of $i$ OFDM sample intervals. Due to the uncorrelated multipaths, the correlation matrix of the channel impulse response becomes a diagonal matrix with the diagonal elements given by the power delay profile. Then the LMMSE's matrix multiplication (or scalar multiplication on corresponding channel taps) is performed as follows [19], [15]:

$$
\begin{aligned}
\hat{h}_{m m s e}= & \operatorname{diag}\left\{\frac{\sigma_{h_{0}}^{2}}{\sigma_{h_{0}}^{2}+\beta / S N R}, \frac{\sigma_{h_{1}}^{2}}{\sigma_{h_{1}}^{2}+\beta / S N R},\right. \\
& \left.\cdots, \frac{\sigma_{h_{N-1}}^{2}}{\sigma_{h_{N-1}}^{2}+\beta / S N R}\right\} \hat{h}_{L S}
\end{aligned}
$$

where $\operatorname{diag}\left\{a_{0}, a_{1}, \cdots, a_{N-1}\right\}$ is a diagonal matrix with diagonal elements $\left[a_{0}, a_{1}, \cdots, a_{N-1}\right]$. In DFT-based LMMSE approach, the first symbol is composed of all pilot tones of equal amplitude and the following data symbols are composed of M-ary QAM symbols on all subcarriers. Then for the initial channel estimation based on the training symbol, $S N R / \beta$ in (26) is replaced by the pilot-to-noise power ratio. For the following channel estimation operated in the decision directed mode, $\beta$ in (26) is given by $\beta=E\left[|X|^{2}\right] E\left[|X|^{-2}\right]$ where $X$ is M-ary QAM symbol. For 64-QAM, $\beta=2.6854$. For MST-LMMSE, $S N R / \beta$ is replaced by its pilot-to-noise power ratio (which is $1 / K$ times that of DFT-based LMMSE due to its pilot ratio of $1 / K$ ).

There is a delicate difference between DFT-based LMMSE and MST-LMMSE. In DFT-based LMMSE, all $N$ samples of $\hat{h}_{L S}$ are corrupted by iid AWGN noise samples of zero mean and variance $\sigma_{t}^{2} / A^{2}$. In MST-LMMSE, the first M samples of $\hat{h}_{L S}$ are corrupted by iid AWGN noise samples of zero mean and variance $K \sigma_{t}^{2} / A^{2}$ and the rest are zeros. Hence, if compared for the training symbol alone, DFT-based LMMSE will have a better channel estimation performance due to the larger pilot power used in channel estimation. But for the following data symbols, DFT-based LMMSE has to use decision directed approach which may degrade the channel estimation performance and BER performance due to the decision directed errors. For MST-LMMSE, the channel estimation and BER performances remain the same for all symbols.

In LMMSE implementation, since multipath channel correlation and SNR are usually unknown at the receiver, some fixed values have to be used for them. In [18], it is suggested to use high dummy SNR value and uniform multipath channel correlation which are robust to the channel correlation mismatch. If high dummy SNR value is used and uniform multipath channel correlation is assumed over guard interval, then LMMSE approach would be similar to [16]'s method. From (26) with high dummy SNR value, the LMMSE approach can be viewed as setting the channel taps with no energy to zeros and bypassing the other taps. Since most practical multipath channels have only a few significant paths (if compared to $N$ ), a suitable way of implementing an approximate LMMSE is choosing a predefined number of most significant taps and setting the others to zero which is the underlying idea of MST method. In complexity aspect, MST-LMMSE estimator still saves one $N$-point IFFT operation if compared to DFT-based LMMSE estimators.

\section{Channel Estimation Performance Evaluation by SIMULATION}

The proposed MST channel estimation is evaluated by computer simulation for two multipath fading channel models, namely Channel-A and Channel-B. The Channel-A is the ATTC (Advanced Television Technology Center) and the Grand Alliance DTV laboratory's ensemble E model whose channel impulse response for the static case is given by

$$
\begin{aligned}
h[n]= & \delta[n]+0.3162 \delta[n-2]+0.1995 \delta[n-17] \\
& +0.1296 \delta[n-36]+0.1 \delta[n-75] \\
& +0.1 \delta[n-137]
\end{aligned}
$$

where unit delay is assumed to be the same as OFDM sample period. The Channel-B is a simplified version of DVB-T channel model $P_{1}$ [20] and its channel impulse response for the static case is given in Table I. In the simulation, the given channel tap gains of both channels represent the standard deviations of the gains of the complex Gaussian random variables.

The OFDM system parameters are as follows: the number of subcarriers $N=8192$, pilot ratio $1 / K=1 / 8$, guard interval ratio $=1 / 8$ and carrier modulation 64-QAM. Perfect synchronization is assumed in order to observe the channel estimation performance alone. The considered methods are 
TABLE I

ChanNel Impulse Response For CHANNEl-B

\begin{tabular}{||c|c|c||}
\hline $\begin{array}{c}\text { Delay } \\
\text { (OFDM samples) }\end{array}$ & Gain & $\begin{array}{c}\text { Phase } \\
\text { (radians) }\end{array}$ \\
\hline 0 & 0.2478 & -2.5694 \\
\hline 1 & 0.1287 & -2.1208 \\
\hline 3 & 0.3088 & 0.3548 \\
\hline 4 & 0.4252 & 0.4187 \\
\hline 5 & 0.4900 & 2.7201 \\
\hline 7 & 0.0365 & -1.4375 \\
\hline 8 & 0.1197 & 1.1302 \\
\hline 12 & 0.1948 & -0.8092 \\
\hline 17 & 0.4187 & -0.1545 \\
\hline 24 & 0.3170 & -2.2159 \\
\hline 29 & 0.2055 & 2.8372 \\
\hline 49 & 0.1846 & 2.8641 \\
\hline & &
\end{tabular}

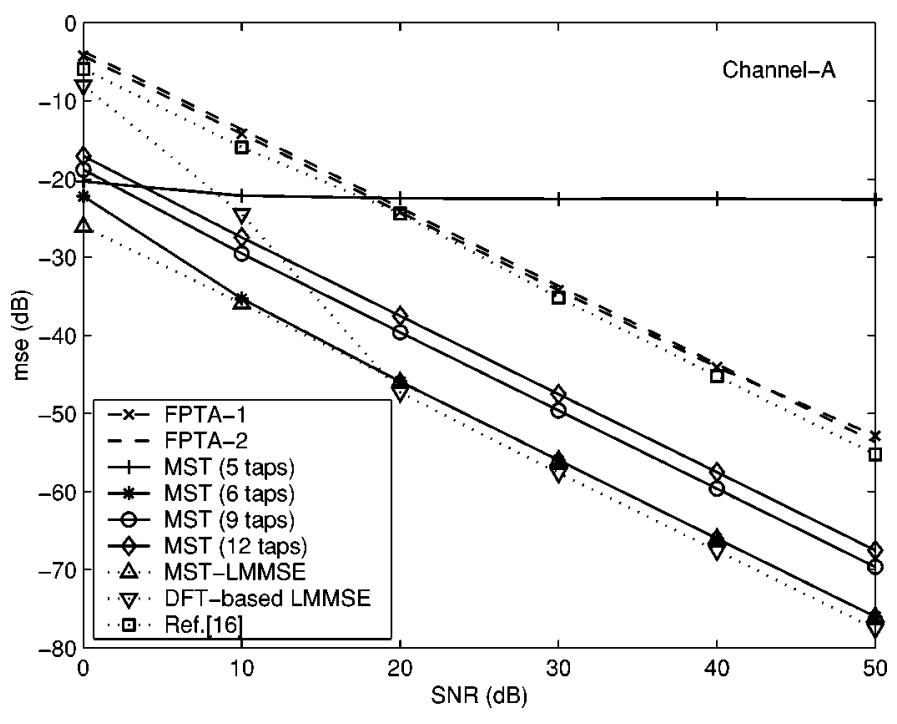

Fig. 3. Channel estimation mean square error ( $m s e)$ in Channel-A.

MST, FPTA-1, FPTA-2, MST-LMMSE, DFT-based LMMSE and [16]. In the last two methods, one OFDM symbol is used for training symbol (pilot tones on all subcarriers) in every $K$ OFDM symbols (i.e., the same pilot ratio $1 / K$ ). In both LMMSE methods, ideal channel correlation and SNR values are used in order to evaluate the relative performance of MST method. In all methods, the same pilot tone symbol of $7+j 7$ signal point in 64-QAM constellation is used. For SNR of 40 and $50 \mathrm{~dB}, 10^{5}$ independent simulation trials are used and for the other SNR values, $10^{4}$ independent trials are used. In our simulation, floating point computation is used. However, it should be noted that due to the limited quantization levels in real hardware implementation, the computation error in real situation may degrade the performance obtained in our simulation.

Fig. 3 shows the mse performances of different channel estimation methods in Channel-A. The required interpolation in FPTA-1 is performed using Matlab's default interp function. Due to some noise reduction of this interpolation, the mse of FPTA-1 is slightly less than FPTA-2. The method of [16] has

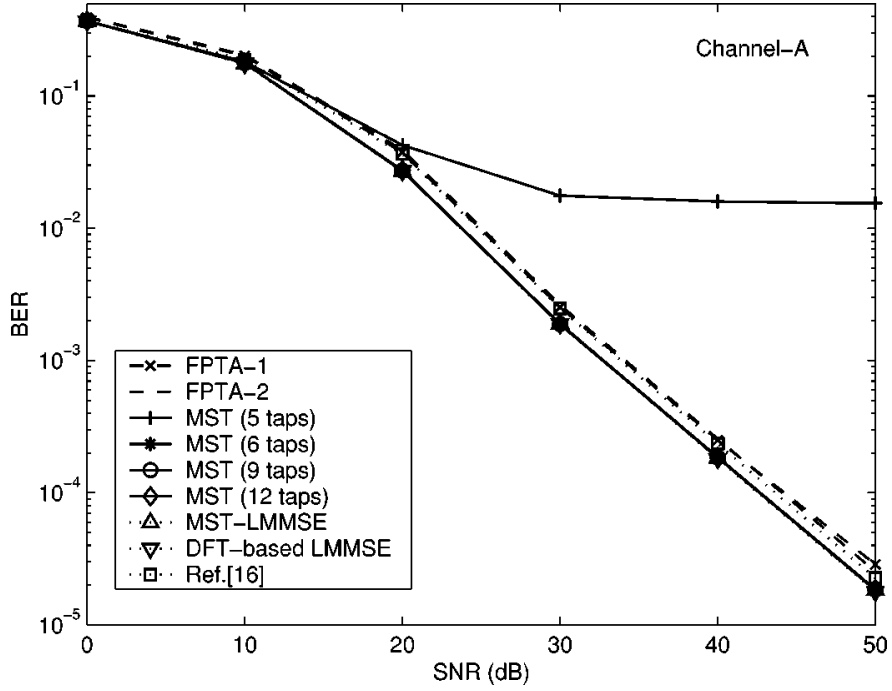

Fig. 4. BER performances of OFDM system with different channel estimation methods in Channel-A.

some improvement over FPTA approaches even though it is associated with decision directed errors. MST method with 6 taps, which is the same as the number of taps in Channel-A, has approximately 18 to $22 \mathrm{~dB}$ mse gain over FPTA approaches. MST with 5 taps shows an irreducible channel estimation error floor caused by missing some of the channel energy. It has a better performance than FPTA approaches for SNR less than $20 \mathrm{~dB}$ since for this SNR region the gain in noise suppression is greater than the loss of channel energy missing. However, for higher SNR region where noise has smaller impact than the channel energy missing, the channel estimation error floor results in a worse performance for MST with 5 taps. For the cases of MST with larger number of taps than the actual channel taps, MST achieves performance gain over FPTA and [16] approaches. The channel estimation performance gains are not as high as MST with 6 taps case due to the additional noise perturbation from the extra taps.

Also shown in Fig. 3 are the performances of MST-LMMSE and DFT-based LMMSE methods. LMMSE's approaches are of similar performance to MST with 6 taps case. For SNR less than $20 \mathrm{~dB}$, DFT-based LMMSE has a worse performance than MST with 6 taps due to the decision directed errors. For higher SNR region, it has a slightly better performance due partly to the fact that its decision directed errors become insignificant and partly to the fact that MST suffers some interference from data part. MST-LMMSE has a slight performance gain over MST with 6 taps case for low SNR region and almost the same performance for high SNR region. This slight performance gain of MST-LMMSE in low SNR region is due to the utilization of (ideal) channel and noise statistics in MST-LMMSE approach by which the selection of channel taps are always correct, whereas in MST approach, the selection of channel taps may not be always correct due to the large noise perturbation.

The actual mse gain of MST method is slightly less than the potential gain of MST method described in previous section since data signal interference to training signal is neglected in the derivation of the potential gain. The actual mse gain is less in low SNR region than in high SNR region. The reason is 
TABLE II

BER For DifFERENT CHANNEl ESTIMATION METHOdS IN CHANNEl-A

\begin{tabular}{||c|c|c|c|c|c|c||}
\hline SNR (dB) & 0 & 10 & 20 & 30 & 40 & 50 \\
\hline FPTA-1 & 0.3926 & 0.2015 & $3.7501 \times 10^{-2}$ & $2.5364 \times 10^{-3}$ & $2.4960 \times 10^{-4}$ & $2.8721 \times 10^{-5}$ \\
\hline FPTA-2 & 0.3957 & 0.2049 & $3.9122 \times 10^{-2}$ & $2.6389 \times 10^{-3}$ & $2.5513 \times 10^{-4}$ & $2.5377 \times 10^{-5}$ \\
\hline MST (5 taps) & 0.3693 & 0.1814 & $4.262 \times 10^{-2}$ & $1.7651 \times 10^{-2}$ & $1.5964 \times 10^{-2}$ & $1.5441 \times 10^{-2}$ \\
\hline MST (6 taps) & 0.3689 & 0.1770 & $2.7049 \times 10^{-2}$ & $1.8693 \times 10^{-3}$ & $1.8358 \times 10^{-4}$ & $1.8309 \times 10^{-5}$ \\
\hline MST (9 taps) & 0.3695 & 0.1776 & $2.7291 \times 10^{-2}$ & $1.8841 \times 10^{-3}$ & $1.8505 \times 10^{-4}$ & $1.8502 \times 10^{-5}$ \\
\hline MST (12 taps) & 0.3700 & 0.1781 & $2.7487 \times 10^{-2}$ & $1.8958 \times 10^{-3}$ & $1.8598 \times 10^{-4}$ & $1.8574 \times 10^{-5}$ \\
\hline MST-LMMSE & 0.3689 & 0.1753 & $2.7023 \times 10^{-2}$ & $1.9081 \times 10^{-3}$ & $1.8309 \times 10^{-4}$ & $1.8299 \times 10^{-5}$ \\
\hline DFT-based LMMSE & 0.3670 & 0.1762 & $2.7051 \times 10^{-2}$ & $1.8911 \times 10^{-3}$ & $1.8052 \times 10^{-4}$ & $1.7851 \times 10^{-5}$ \\
\hline Ref. [16] & 0.3711 & 0.1887 & $3.6529 \times 10^{-2}$ & $2.4789 \times 10^{-3}$ & $2.3445 \times 10^{-4}$ & $2.2593 \times 10^{-5}$ \\
\hline
\end{tabular}

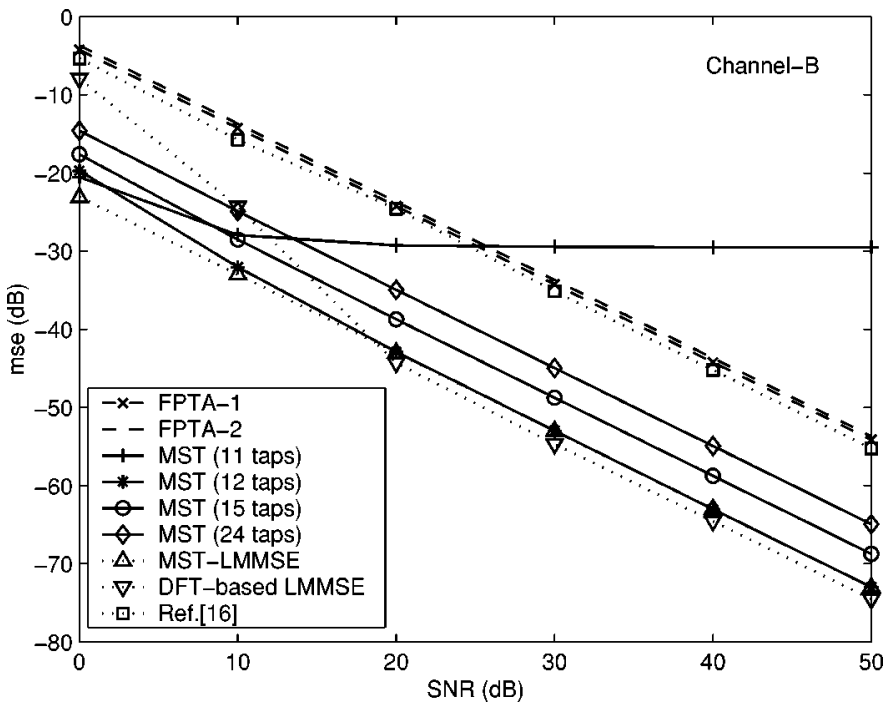

Fig. 5. Channel estimation mean square error (mse) in Channel-B.

that most significant channel taps selection would more likely choose some taps with no channel energy at low SNR region than at high SNR region, resulting in less mse gain at low SNR region.

In Fig. 4, the BER performances in Channel-A are presented for different channel estimation methods. Due to the channel estimation error floor and the BER sensitivity of 64-QAM to channel estimation error, MST with 5 taps case shows a BER floor while the others do not. The method of [16] is slightly better than FPTA approaches as is the case in mse performance. The other MST and LMMSE approaches have better BER performances than FPTA and [16] approaches, but among themselves, the BER performances are just slightly different and hence their BER curves are almost the same. For a better presentation of their slight differences, their BER values are tabulated in Table II. These slight differences can be ascribed to the corresponding different channel estimation performances. It is also noted that when the channel estimation $m s e$ is much smaller than 1/SNR, then some deviation in channel estimation mse does not significantly affect the BER performance since noise is the dominant contributor to BER in this case.

The channel estimation performance and BER performance of the considered methods in Channel-B are plotted in Figs. 5 and 6 respectively and the BER values are given in Table III for the sake of clarity. The performances are of the same trend as in

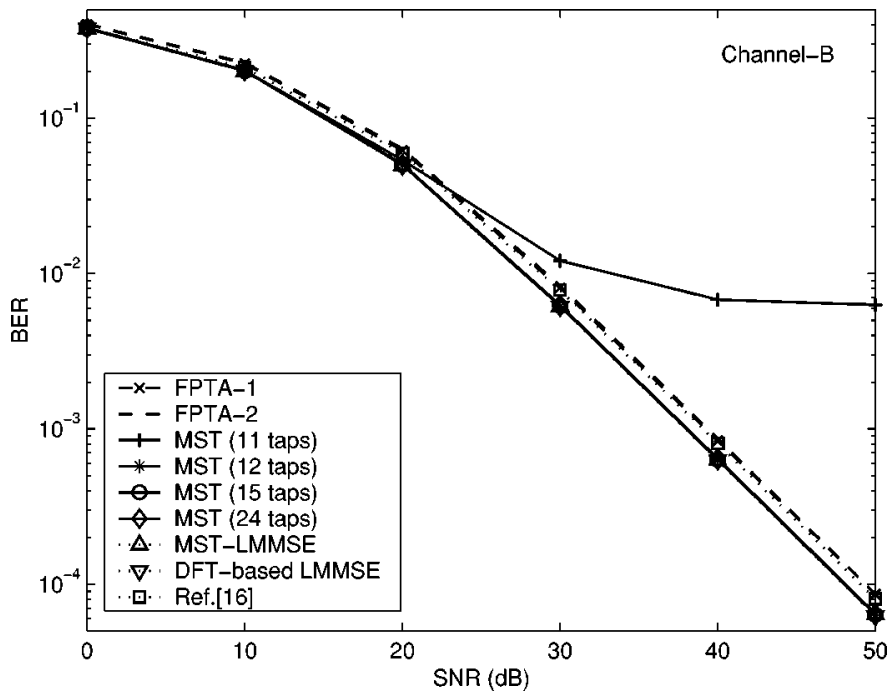

Fig. 6. BER performances of OFDM system with different channel estimation methods in Channel-B.

Channel-A. It is also noted that the channel estimation and BER performances in Channel-B is worse than Channel-A except for the case of missing some of the channel energy (i.e., MST with 5 taps in Channel-A and MST with 11 taps in Channel-B). This exception is due to the larger percentage of the channel energy missed in Channel-A than in Channel-B.

From the simulation results, it is clear that the number of MST taps $J$ should not be smaller than the number of actual channel taps. On the other hand, using more taps may slightly degrade the BER performance due to the more noise perturbation. However, even up to double of the number of actual channel taps, MST has almost the same BER performance as using the number of actual channel taps. Hence, a suitable choice for $J$ might be double of the (designed) number of channel taps for the considered channel environment.

Another way of selecting MST taps by threshold decision is also evaluated in Channel-B and the channel estimation mse and BER results are shown in Figs. 7 and 8 respectively. The performance of MST with 12 taps is also included for reference. It is observed that the suitable choice of threshold also depends on the operating SNR, as expected. The results also indicate that almost the same performance as MST with 12 taps case can be obtained if the threshold for an operating SNR is set within approximately 20 to $23 \mathrm{~dB}$ below the operating $1 / S N R$. This low 
TABLE III

BER FOR DiFFERENT CHANNEL EsTIMATION METHODS IN CHANNEL-B

\begin{tabular}{||c|c|c|c|c|c|c||}
\hline SNR (dB) & 0 & 10 & 20 & 30 & 40 & 50 \\
\hline FPTA-1 & 0.4014 & 0.2250 & $6.1986 \times 10^{-2}$ & $8.0725 \times 10^{-3}$ & $8.3867 \times 10^{-4}$ & $8.6296 \times 10^{-5}$ \\
\hline FPTA-2 & 0.4041 & 0.2283 & $6.3793 \times 10^{-2}$ & $8.3684 \times 10^{-3}$ & $8.6701 \times 10^{-4}$ & $8.6831 \times 10^{-5}$ \\
\hline MST (11 taps) & 0.37828 & 0.2019 & $5.3833 \times 10^{-2}$ & $1.2092 \times 10^{-2}$ & $6.7836 \times 10^{-3}$ & $6.2976 \times 10^{-3}$ \\
\hline MST (12 taps) & 0.3785 & 0.2011 & $4.9783 \times 10^{-2}$ & $6.1269 \times 10^{-3}$ & $6.2924 \times 10^{-4}$ & $6.3026 \times 10^{-5}$ \\
\hline MST (15 taps) & 0.3790 & 0.2017 & $5.0082 \times 10^{-2}$ & $6.1707 \times 10^{-3}$ & $6.3410 \times 10^{-4}$ & $6.3421 \times 10^{-5}$ \\
\hline MST (24 taps) & 0.3805 & 0.2032 & $5.0749 \times 10^{-2}$ & $6.2714 \times 10^{-3}$ & $6.4442 \times 10^{-4}$ & $6.4503 \times 10^{-5}$ \\
\hline MST-LMMSE & 0.3781 & 0.2011 & $4.9847 \times 10^{-2}$ & $6.1509 \times 10^{-3}$ & $6.3000 \times 10^{-4}$ & $6.3806 \times 10^{-5}$ \\
\hline DFT-based LMMSE & 0.3775 & 0.2018 & $4.969 \times 10^{-2}$ & $6.1261 \times 10^{-3}$ & $6.2774 \times 10^{-4}$ & $6.2568 \times 10^{-5}$ \\
\hline Ref. [16] & 0.3809 & 0.2123 & $5.9644 \times 10^{-2}$ & $7.7969 \times 10^{-3}$ & $8.0554 \times 10^{-4}$ & $8.0195 \times 10^{-5}$ \\
\hline
\end{tabular}

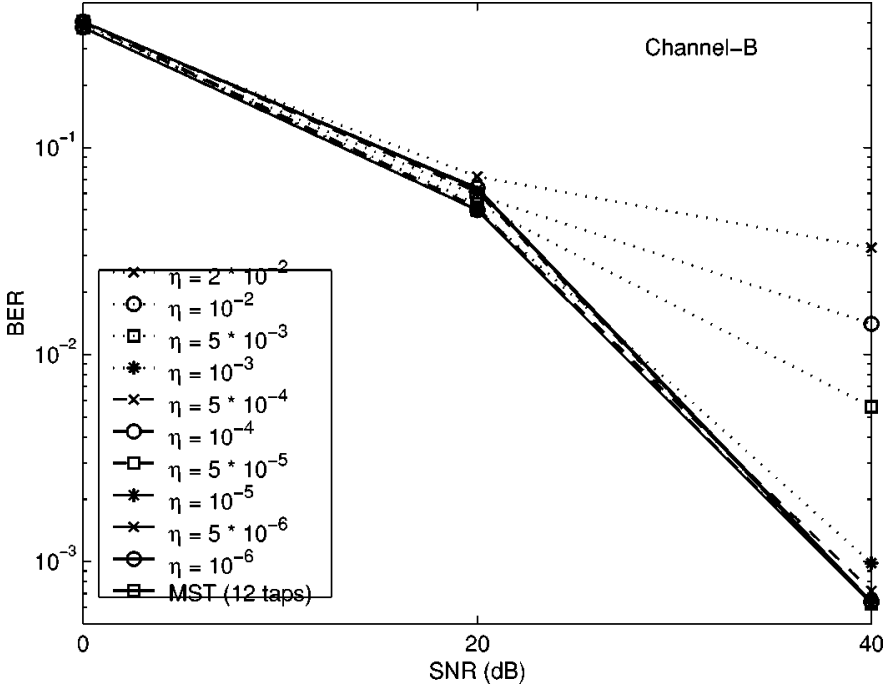

Fig. 7. Channel estimation mean square error (mse) for MST with threshold setting $\eta$.

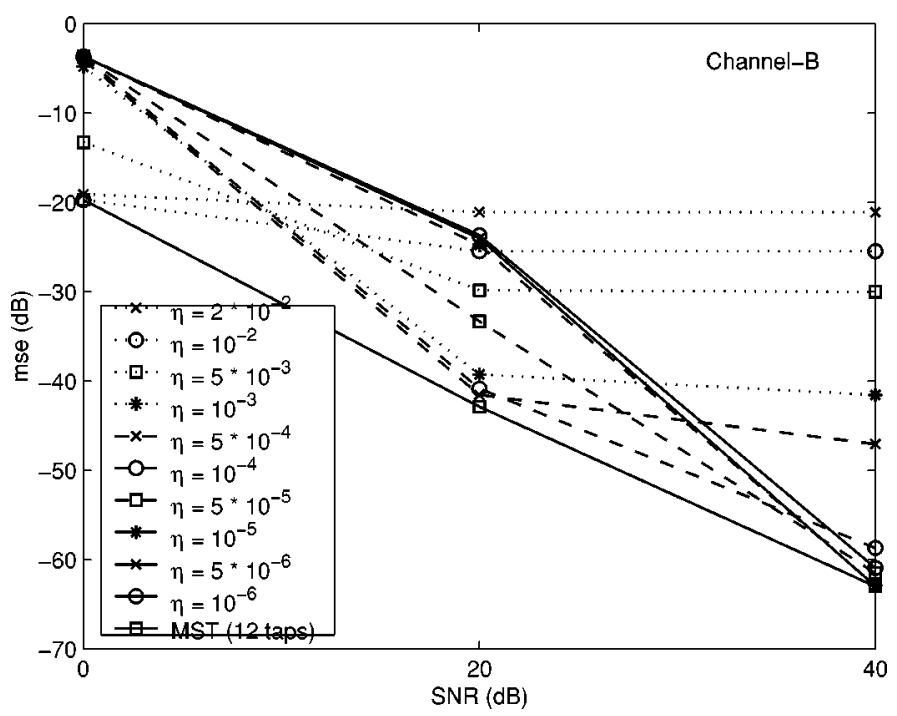

Fig. 8. BER performance of MST with threshold setting $\eta$.

value of the threshold in ensuring no channel energy miss is also due to the gain variations in the channel taps.

It is also remarked that for relatively slow fading environments, the decision feedback approaches such as [16] and
LMMSE can increase spectrum efficiency by using smaller pilot ratio. Similarly, MST approach can increase the spectrum efficiency by not inserting pilot tones in every OFDM symbol. For this case, the decision feedback approach as used in MST's dual form DFT-based approach can easily be applied for pure data symbols.

\section{CONCLUSION}

In this paper, intra-symbol time-domain averaging approaches for time-domain channel estimation of OFDM system are investigated. As an approximation to LMMSE type estimator, a time-domain approach, called MST, is proposed where intra-symbol time-domain averaging and most significant taps selection are applied. The similarities and differences of the proposed MST method versus DFT-based LMMSE methods are discussed. Two approaches for MST taps selection are described where the first uses a fixed number of taps while the second chooses all taps above a threshold. The simulation results suggest that a suitable choice for the fixed number of MST taps is using double of the (designed) number of channel taps for the considered channel environment. And a suitable choice of the threshold is within the range of approximately 20 to $23 \mathrm{~dB}$ below the operating $1 / S N R$. In terms of BER performance in multipath fading channels, MST without channel energy missing and LMMSE approaches have almost the same performance which is better than the other considered approaches. In terms of complexity, MST approach keeps minimum complexity among the considered methods.

\section{ACKNOWLEDGMENT}

The authors would like to thank the editor and anonymous reviewers for the helpful comments and suggestions that improved the quality of this paper.

\section{REFERENCES}

[1] R. W. Chang, "Synthesis of band-limited orthogonal signals for multichannel data transmission," Bell Syst. Tech. Journal, vol. 45, Dec. 1966.

[2] L. J. Cimini, Jr., "Analysis and simulation of a digital mobile channel using orthogonal frequency-division multiplexing," IEEE Trans. Commun., vol. 33, no. 7, pp. 665-675, July 1985.

[3] J. A. C. Bingham, "Multicarrier modulation for data transmission: An idea whose time has come," IEEE Commun. Mag., vol. 28, no. 5, pp. 5-14, May 1990

[4] Asymmetric digital subscriber line (ADSL) metallic interface, American National Standards Institute (ANSI), Dec. 1995. 
[5] P. Shelswell, "The COFDM modulation system: The heart of digital audio broadcasting," Elec. Commun. Eng. Journal, pp. 127-136, June 1995.

[6] U. Reimers, "Digital video broadcasting," IEEE Communications Magazine, vol. 36, no. 6, pp. 104-110, June 1998.

[7] M. Uehara, M. Takada, and T. Kuroda, "Transmission scheme for the terrestrial ISDB system," IEEE Trans. on Consumer Electronics, vol. 45, no. 1, pp. 101-106, Feb. 1999.

[8] R. van Nee and G. Awater, "New high-rate wireless LAN standards," IEEE Communications Magazine, pp. 82-88, Dec. 1999.

[9] N. Morinaga and A. Hashimoto, "Technical trend of multimedia mobile and broadband wireless access systems," IEICE Trans. Commun., vol. E82-B, no. 12, pp. 1897-1905, Dec. 1999.

[10] Y. Li, N. Seshadri, and S. Ariyavisitakul, "Channel estimation for OFDM systems with transmitter diversity in mobile wireless channels," IEEE Journal on Select. Areas in Comms., vol. 17, no. 3, pp. 461-470, Mar. 1999.

[11] Y. Li, L. J. Cimini, Jr., and N. R. Sollenberger, "Robust channel estimation for OFDM systems with rapid dispersive fading channels," IEEE Trans. on Comms., vol. 46, no. 7, pp. 902-915, July 1998.

[12] M. Speth, S. Fechtel, G. Fock, and H. Meyr, "Broadband transmission using OFDM: System performance and receiver complexity," in International Zurich Seminar on Broadband Communications, Zurich, Switzerland, Feb. 1998, pp. 99-104

[13] P. Hoeher, "TCM on frequency selective land mobile fading channel," in Tirrenia International Workshop on Digital Communications, Sept. 1991.

[14] F. Classen, M. Speth, and H. Meyr, "Channel estimation units for an OFDM system suitable for mobile communications," in Mobile Kommunikation: ITG-Fachbericht, Munchen, Berlin, Sept. 1995.

[15] J.-J. van de Beek, O. Edfors, M. Sandell, S. K. Wilson, and P. O. Brjesson, "On channel estimation in OFDM systems," in Vehicular Technology Conf., 1995, pp. 815-819.

[16] A. Chini, Y. Wu, M. El-Tanany, and S. Mahmoud, "Filtered decision feedback channel estimationfor OFDM-based DTV terrestrial broadcasting system," IEEE Trans. on Broadcasting, vol. 44, no. 1, pp. 2-11, Mar. 1998.

[17] C. S. Yeh and Y. Lin, "Channel estimation using pilot tones in OFDM systems," IEEE Trans. on Broadcasting, vol. 45, no. 4, pp. 400-409, Dec. 1999.
[18] O. Edfors, M. Sandell, J.-J. van de Beek, S. K. Wilson, and P. O. Borjesson, "OFDM channel estimation by singular value decomposition," IEEE Trans. Commun., vol. 46, pp. 931-939, July 1998.

[19] S. M. Kay, Fundamentals of Statistical Signal Processing - Estimation Theory: Prentice Hall PTR, 1993.

[20] Digital video broadcasting (DVB): Framing, channel coding and modulation for digital terrestrial television, DRaft ETSI EN300 744 V1.3.1 (2000-08).

H. Minn received his B.E.(Electronics) degree with honors from Yangon Institute of Technology, Myanmar, in 1995, and M.Eng. (Telecommunications) degree from Asian Institute of Technology (AIT), Thailand, in December 1997. He worked as a laboratory supervisor at Telecommunications Program, AIT, for one year. Since 1999, he has been a research assistant in the Department of Electrical and Computer Engineering at the University of Victoria, Canada. Currently, he is a Ph.D. student at University of Victoria. His research interests include wireless communications, OFDM, synchronization, channel estimation, equalization, modulation and error control coding.

Vijay K. Bhargava received the B.Sc., M.Sc., and Ph.D. degrees from Queen's University, Kingston, Canada in 1970, 1972 and 1974 respectively. Currently, he is a Professor of Electrical and Computer Engineering at the University of Victoria. He is a co-author of the book Digital Communications by Satellite (New York: Wiley, 1981) and co-editor of the IEEE Press Book Reed-Solomon Codes and Their Applications. He is an Editor-in-Chief of WIRELESS PERSONAL COMMUNICATION, a Kluwer Periodical. His research interests are in multi-media wireless communications.

Dr. Bhargava is very active in the IEEE and is currently the President of the IEEE Information Theory Society. He was Co-chair for IEEE ISIT'95, Technical Program Chair for IEEE ICC'99 and is the Chair of IEEE VTC'2002 Fall.

Dr. Bhargava is a Fellow of the B.C. Advanced Systems Institute, Engineering Institute of Canada (EIC), IEEE and the Royal Society of Canada. He is a recipient of the IEEE Centennial Medal (1984), IEEE Canada's McNaughton Gold Medal (1995), the IEEE Haraden Pratt Award (1999) and the IEEE Third Millennium Medal (2000). 